Trabajos Originales

\title{
El método de clasificación de mortinatos “Condición Obstétrica Relevante de la Muerte Fetal" (CORM) reduce la frecuencia de muerte fetal de causa inexplicable.
}

\author{
Drs.Alfredo Ovalle $S^{1}$, Oscar Valderrama $C^{1}$, Jorge S. Alvarado Ch ${ }^{1}$, Elena \\ Kakarieka W ${ }^{2}$.
}

1 Servicio y Departamento de Obstetricia, Ginecología y Neonatología, Hospital Clínico San Borja Arriarán. Facultad de Medicina, Universidad de Chile.

2 Servicio de Anatomía Patológica, Hospital Clínico San Borja Arriarán.

\section{RESUMEN}

Introducción: La muerte fetal (MF) es el principal contribuyente de la mortalidad perinatal. Objetivo: Analizar la eficiencia del método de clasificación de mortinatos, condición obstétrica relevante de la MF (CORM). Métodos: Estudio cohorte retrospectivo, de las MF ocurridas en la Maternidad del Hospital San Borja Arriarán durante 10 años. Los datos provienen de los informes de las auditorías de mortinatos. Se aplicó el método de clasificación CORM, que usa la biopsia placentaria, los datos clínicos y de laboratorio analizados por un obstetra y un patólogo. Resultados: Ocurrieron 56.130 nacimientos y 479 MFs. Tasa de MF, 8.5 por 1000 nacimientos. Se identificaron el $93.5 \%$ de las condiciones obstétricas de MF y $6.5 \%$ fueron inexplicables. Las más frecuentes fueron: infección bacteriana ascendente (IBA) $24.9 \%$, anomalía congénita $18.0 \%$, y patología placentaria $14.0 \%$. La restricción del crecimiento fetal intrauterino (RCIU) se presentó en el $49.7 \%$ de los mortinatos, la mayoría $93.7 \%$, fue secundaria a condición obstétrica primaria. La asfixia durante el parto fue el $0.8 \%$ de los mortinatos y se presentó en embarazos de término. El parto prematuro (PP) representó el $80 \%$ de los casos de MF. La condición más frecuente asociada con PP fue IBA (38.5\% y 38.2\% de las MF menores de 30 semanas y menores de 1000 gramos respectivamente). Conclusiones: El método clasificación de mortinatos CORM, es más eficiente que los métodos convencionales en detectar la causa de MF porque identifica la mayoría de las condiciones obstétricas responsables de la MF. Es reproducible, requiere estudio histopatológico de la placenta y no de autopsia fetal. Permite elaborar guías de prevención por causa.

PALABRAS CLAVE: Sistemas de clasificación, muerte fetal, prevención.

\section{SUMMARY}

Introduction: Stillbirth is the main contributor to perinatal mortality. Aim: To analyze the efficiency of the method for classification of fetal death (FD), "obstetric condition relevant to the death (OCRD). Methods: Retrospective cohort study of the FD that occurred in the Maternity Hospital of San Borja Arriarán for ten years. The data comes from the reports of the stillborn audits. We applied the classification method OCRD, which uses placental biopsy, clinical and laboratory data analyzed by a single obstetrician and a single pathologist. Results: 56,130 births and 479 FD occurred. FD rate, 8.5 per 1000 births. We identified $93.5 \%$ of the obstetric conditions of FD and $6.5 \%$ were unexplained. The most frequent were: ascending bacterial infection (ABI) $24.9 \%$, congenital anomaly $18.0 \%$, and placental pathology $14.0 \%$. The intrauterine fetal growth restriction (IUGR) was present in $49.7 \%$ of the stillbirths, the majority $93.7 \%$, was secondary to primary obstetric condition. Asphyxia during delivery was $0.8 \%$ of the stillbirths and occurred in term pregnancies. Preterm birth (PB) represented $80 \%$ of FD cases. The most frequent condition associated with $\mathrm{PB}$ was $\mathrm{ABI}(38.5 \%$ and $38.2 \%$ of the FD less than 30 weeks and less 
than 1000 grams respectively). Conclusions: The OCRD stillbirth classification method is more efficient than conventional methods in detecting the cause of MF because it identifies most of the obstetric conditions responsible for FD. It is reproducible, requires histopathological study of the placenta and not fetal autopsy. It allows developing guidelines of prevention for cause.

KEY WORDS: Classification systems, stillbirth, prevention.

\section{INTRODUCCIÓN}

La muerte fetal (MF) es el mayor contribuyente de la mortalidad perinatal en el mundo. En la mayoría de los países, la tasa de mortinatalidad es igual o superior a la tasa de mortalidad neonatal, con cerca de 3 millones de MF en el tercer trimestre $(1,2)$. Si se agregan los mortinatos del segundo trimestre, esta cifra se incrementa a más de 5 millones por año en todo el mundo (3).

En el hospital Clínico San Borja Arriarán (HCSBA) la tasa de MF aumentó desde 6.8 en 2008, hasta 10.2 por mil nacidos en 2016 con participación de la migración que ha crecido durante los últimos años (Sistema Informático Perinatal. OPS/OMS CLAP, datos no publicados).

Los diferentes sistemas de clasificación de mortinatos actuales que ocupan la causa final o primaria de MF determinada preferentemente por la autopsia fetal, no son suficientemente eficientes, porque no identifican la causa de MF en la mayoría de los casos lo que impide hacer normas de prevención adecuadas que permitan reducir este resultado adverso(4-13).

Reportan con frecuencia muertes inexplicables y restricción del crecimiento intrauterino (RCIU) de origen no precisado (4-13). Además, emplean la asfixia fetal $(3,14$,$) y el parto prematuro (15,16)$ como causas primarias de MF, cuyos orígenes variados dificultan la clasificación de la MF y la elaboración de normas de prevención. Estos argumentos posiblemente han participado para que las tasas de MF permanezcan elevadas en el mundo $(5,10,11)$.

Para priorizar las intervenciones y mejorar los resultados perinatales, es necesario tener un método que identifique la condición obstétrica que origina los eventos que conducen a la MF (aquélla que en su ausencia no ocurre el óbito), probablemente la mejor forma de prevenir este resultado adverso $(10,17,18)$.

En el HCSBA desde hace algunos años ocupamos el método, "condición obstétrica relevante de la muerte" (CORM), basándonos en los antecedentes obstétricos y biopsia placentaria (19). El análisis histopatológico de la placenta permite conocer el daño de este órgano causado por la condición obstétrica originaria de la MF $(12,13,16,19)$. Estas lesiones placentarias, habitualmente específicas, permiten aclarar cuál es la causa que participa en la muerte intrauterina $(12,13,16,19)$. Su conocimiento nos ayuda a elaborar estrategias de prevención por causa para evitar mortinatos futuros (16).

El objetivo de este trabajo es analizar la eficiencia del método de clasificación de mortinatos, condición obstétrica relevante de la MF, que permite identificar la causa originaria de la mortinatalidad.

\section{MÉTODOS}

Los datos del estudio provienen de la base de datos de los informes de auditoría de muerte fetal, realizados por uno de los autores $(A O)$, entre el $1 \%$ de enero de 2008 y el 31 de diciembre de 2017 en el Servicio de Obstetricia, Ginecología y Neonatología del Hospital San Borja Arriarán.

Datos incluidos: fecha del nacimiento, edad gestacional, datos maternos (edad, paridad, clínicos, laboratorio, ultrasonográficos), cálculo del percentil por edad gestacional, sexo y peso al nacer del recién nacido (RN), detalles de embarazo para determinar la condición de muerte fetal y biopsia placentaria. El cálculo del percentil se hizo mediante las curvas Pittaluga-Alarcón $(20,21)$, curvas antropométricas de población chilena. Se estimó como RCIU el peso al nacer < del percentil 10. Se incorporaron embarazos desde las 22 semanas con edad gestacional segura, calculada por ultrasonografía precoz.

El Comité de Ética Científico del Servicio de Salud Metropolitano Central aprobó la realización de este trabajo.

\section{Clasificación de las muertes fetales}

Nuestro sistema de clasificación se basó en métodos propuestos anteriormente $(12,13,16)$ que permitieron desarrollar el sistema CORM(9) (condición obstétrica relevante de la muerte) utilizando los datos obstétricos (clínicos y de laboratorio) y los hallazgos histopatológicos placentarios. Este método nos permitió conocer la condición obstétrica originaria de la muerte $(5,10,17)$. 
Con dos o más condiciones presentes, se seleccionó aquélla de mayor importancia asociada con la muerte. El estudio histopatológico fue realizado por un patólogo (EK).

\section{A) Condición materna}

Enfermedad

1. Síndrome hipertensivo (SH): PE, hipertensión arterial crónica (HAC), con algunas condiciones clínicas: DPPNI, síndrome de HELLP, RCIU, asociado con algunos hallazgos placentarios: infarto vellositario, arteriopatía de vasos deciduales, hematoma retroplacentario, hemorragia intervellositaria, endovasculitis hemorrágicas de vasos fetales, vasculopatía fetal trombótica, trombosis intervellositaria, maduración acelerada $(22,23)$.

2. Diabetes: Mellitus (DM) o gestacional (DG), asociada con algunos hallazgos placentarios: edema e inmadurez vellositaria, maduración vellositaria retardada, vellosidades avasculares, arteriopatía fetal trombótica, infarto vellositario, hematoma retroplacentario, hemorragia intervellositaria, corangiosis(24).

3. Obesidad: Índice de masa corporal $>30 \mathrm{~kg} / \mathrm{m} 2$, con o sin hipoxia crónica (RCIU) y algunos hallazgos placentarios: inmadurez, retraso madurez y edema vellositario, vellosidades avasculares, arteriopatía fetal trombótica, depósito masivo de fibrinoide perivellositario, infarto del lecho placentario, hemorragia y hematoma retroplacentario $(25,26)$.

4. Drogas: Consumo prolongado de cocaína, pasta base, marihuana, tolueno, alcohol, tabaco y otras durante el embarazo, RCIU severo, con o sin DPPNI y con hallazgos placentarios: infarto vellositario, hemorragia y hematoma retroplacentario $(27,28)$.

5. Lupus eritematoso sistémico (LES): Cuadro clínico y de laboratorio compatibles (29).

6. Colestasia intrahepática del embarazo (CIE): Cuadro clínico y de laboratorio compatibles (30).

7. Isoinmunización: por anticuerpos del sistema Rh (anticuerpo anti-D, anti-E), del sistema LMN (anticuerpo anti-M) y por otros anticuerpos (31).

8. Otras, Trombofilia: Cuadro clínico de trombosis (de extremidades 0 tromboembolismo pulmonar), anticuerpos antifosfolípidos positivos, con DPPNI, con o sin RCIU y con hallazgos placentarios: hemorragia y hematoma retroplacentarios, infartos vellositarios, depósito de fibrina intervellositaria, vasculopatía fetal trombótica y trombosis intervellositaria (32).

Otras enfermedades: hipertiroidismo, enfermedad renal crónica, embolía de líquido amniótico, trauma por accidentes, púrpura de Schönlein-Henoch, epilepsia, shock séptico, muerte materna, aneurisma, PTI, enfermedad de Steinert, y otras.

Infección

Ascendente

9. Bacteriana (IBA): Embarazo con algunas condiciones clínicas: rotura prematura de membranas (RPM), corioamnionitis clínica, sangrado vaginal con desprendimiento amniocorial, infección cérvicovaginal (ICV) por Streptococcus Grupo B(SGB), infección del tracto urinario (ITU), DPPNI, cérvix $<15 \mathrm{~mm}$ medido por ultrasonografía, membranas prolapsadas bajo el orificio cervical externo $(16,32-35)$ y con hallazgos placentarios agudos: corioamnionitis histológica $(\mathrm{CH})$, funisitis, intervellositis (22,33-37).

10. Micótica: Hallazgos placentarios agudos: corioamnionitis, funisitis, intervellositis por candida sp.

Transplacentaria

11. Viral: Infección viral materna, con o sin RCIU, con 0 sin hidrops fetal y hallazgos placentarios: vellositis crónica, con o sin necrosis, vellosidades con cambios citopáticos sugerentes de infección viral, intervellositis crónica, corioamnionitis crónica $(22,32,34)$.

12. Sífilis: Infección materna, con o sin infección congénita, con o sin hidrops fetal y con hallazgos placentarios: hidrops placentario, vellositis crónica, arteritis obliterante $(21,33,35)$.

13. Listeria monocytogenes: Infección materna febril por L. monocytogenes aislada en sangre materna $\mathrm{y} / \mathrm{o}$ placenta $\mathrm{y}$ hallazgos placentarios: vellositis, perivellositis abscedada, microabscesos en vellosidades, corioamnionitis, funisitis $(33,35)$.

14. Enfermedad periodontal: Diagnóstico odontológico de periodontitis generalizada, severa y asociada con vellositis, intervellositis aguda en los hallazgos placentarios (38).

15. Otras

B) Condición fetal

Anomalía congénita

1. Cromosómicas $(11,39)$ (con malformaciones múltiples habitualmente). Síndrome de Turner (XO), trisomías 13,18 , y 21 , confirmadas por cariograma realizado en líquido amniótico o sangre fetal y otros defectos cromosómicos sugeridos por hallazgos placentarios: $v$ vellosidades inmaduras con cambios hidrópicos, inclusiones trofoblásticas, trofoblasto hipoplásico, y calcificaciones de membranas basales

2. No cromosómicas (malformaciones de un órgano o sistema). De causa multifactorial, por factores ambientales, enfermedades maternas, agentes infecciosos, físicos, químicos, uso de medicamentos o mecánicos. 
RCIU. 3. De condición no precisada.

Hidrops. 4. De condición no precisada.

Embarazo múltiple (monocorial biamniótico) 5 . Síndrome de Transfusión feto-fetal (STFF). 6. RCIU selectiva.

C) Condición placentaria

Patología placentaria

1. DPPNI primario: Separación de la placenta del útero, en ausencia de patología materno-fetal y con lesiones placentarias: hematoma y hemorragia retroplacentaria, hemorragia subcorial, infartos vellositarios, trombosis intervellositaria (12,33,39-41).

2. Alteraciones circulatorias maternas y fetales y RCIU: Ausencia de patología materno-fetal, con RCIU, con o sin DPPNI, con lesiones placentarias: arteriopatía fetal trombótica, trombosis intervellositaria y arterial fetal, depósito aumentado de fibrinoide perivellositario, infartos vellositarios, hemorragia y hematoma retroplacentario (22,39-41).

3. Deciduitis, vellositis crónica y RCIU: Ausencia de patología materno-fetal, con RCIU y con lesiones placentarias: deciduitis crónica linfoplasmocitaria, corioamnionitis crónica, perivellositis y vellositis crónica, trombosis intervellositaria $(22,42)$.

4. Otras y RCIU: Ausencia de patología maternofetal, con RCIU y con lesiones placentarias: edema vellositario, corangiosis.

5. Patología del cordón umbilical: Circular, nudo, trombosis y/o ruptura, hiperrotación, procidencia, hematoma, inserción velamentosa (39-41).

D) Condición uterina

Malformación uterina, trauma del parto, parto provocado, rotura uterina y ausencia de patología materno-fetal (22).

E) Asfixia durante el parto de término

Embarazo sin patología materna, fetal, placentaria y asfixia por atención obstétrica deficiente.

F) No clasificable, no precisable

Casos en que los antecedentes obstétricos e histopatológicos placentarios no son suficientes para aclarar la condición asociada a la muerte fetal (12).

Análisis estadístico de los datos. Expresión de resultados

Estudio descriptivo. Los resultados se expresan en términos de frecuencia (porcentual) o de medias con su respectivo error estándar. Frecuencia, de la condición obstétrica identificada como responsable de la MF, de la condición obstétrica no identificable de MF y de las condiciones causantes de RCIU, del PP y de asfixia.

Resultados
Este estudio incluyó 56.130 nacimientos, el 75.3\% (42.261) de madres chilenas y el 24,7\% (13.869) de madres extranjeras. Los mortinatos fueron $479(8.5$ por 1000 nacimientos), 384(9.1 por 1000 nacimientos) de madres chilenas y 95(6.8 por 1000 nacimientos) de madres extranjeras.

En la tabla 1 se presenta la Clasificación de la MF según Método CORM.

En la figura 1 se mencionan las tasas de mortalidad fetal en el HCSBA total y por nacionalidad de las madres en el período 2008-2017. Tasa de MF, 8.5 por 1000 nacimientos.

Se identificaron el $93,5 \%$ de las condiciones obstétricas asociadas con la MF y la proporción de mortinatos inexplicables fue $6,5 \%$ de los casos. Las condiciones más frecuentes de MF fueron: a) IBA, $24.9 \%$ (119/479). La IBA se presentó en el $38.5 \%$ (89/231) de los menores de 30 semanas y en el $38.2 \%$ (76/199) de los menores de 1000 gramos. El 74.8\% (89/119) de la MF por IBA son menores de 30 semanas y el 63.9\% (76/119) son menores de 1000 gramos; b)Anomalía congénita 18.0\%(86/479), cromosómica 57 casos: trisomías, 13(2), 18(12), 21(7), síndrome de Turner(7), otras malformaciones múltiples sin cariograma(29) y no cromosómicas(29); c)Patología placentaria 14.0\% (67/479), DPPNI(22), placenta previa(2), patología placentaria (43); d)SH 12,6\% (60/479), PE(47), HAC(13); e)Otra enfermedad materna 6.9\%(33/479), consumo drogas(6), LES(5), CIE(3), isoinmunización(3), trombofilia(3), trauma por accidente(2), hipertiroidismo(2) y enfermedad renal crónica, embolía de líquido amniótico, púrpura de Schönlein-Henoch, epilepsia, shock séptico, muerte materna, aneurisma, PTI, enfermedad de Steinert, 1 caso c/u; f)Embarazo múltiple 3.5\% (17/479, STFF(11), RCIU selectiva(6); g)Diabetes 3.3\% (16/479), DM(8), DG(8); h) Infección transplacentaria $3.1 \%$ (15/479), síflis(4), virales varias(4), $L$. monocytogenes(3), parvovirus $\mathrm{B} 19(2), \quad \operatorname{CMV}(1)$, enfermedad periodontal(1)

La asfixia durante el parto de término por atención obstétrica deficiente fue el $0,4 \%(2 / 479)$ de las muertes. Las demás asfixias se incluyeron en las situaciones obstétricas originarias de MF.

La RCIU se presentó en 49.7\% (238/497) de los mortinatos. El 93.7\% (223/238) fue secundaria a la condición obstétrica identificada como relevante de la muerte. El 6.3\% (15/238) se clasificó como RCIU de condición no precisada (Tabla 2).

En la Tabla 3 se analizan las características de la población en estudio. Antes de las 37 semanas ocurrió $80 \%$ de los mortinatos (383), antes de las $34,66.0 \%$ 
(316) y 48,2\% (231) antes de las 30 semanas. El $56.8 \%$ de los óbitos pesó menos de $1.500 \mathrm{~g}$ al nacer, $78,5 \%$ menos de 2.500 g y $21,5 \%$ más de $2.500 \mathrm{~g}$. La IBA $26.1 \%$ y la anomalía congénita $18.8 \%$, fueron las condiciones obstétricas más frecuentes en los PP. Hubo 11 casos con restricción de crecimiento muy severa al nacer (< de $400 \mathrm{~g}$ ): 5 casos de $\mathrm{SH}, 3$ patologías placentarias y 3 de otras causas.

Discusión

De acuerdo a los resultados de este estudio el método de clasificación de mortinatos CORM, que usa la biopsia placentaria, los datos clínicos y de laboratorio, es más eficiente que los sistemas de clasificación de MF tradicionales, en identificar la causa o condición obstétrica originaria de muerte.

En esta serie la condición obstétrica relevante de la MF se conoció en el $93.5 \%$ de los casos y en el $6.5 \%$ las causas fueron inexplicables y muy superior a resultados obtenidos con otras clasificaciones que encuentran $62 \%$ de muertes explicables (4) y $15.2 \%$ de muertes inexplicables (10). Las condiciones más frecuentes de MF fueron IBA (24.9\%), anomalía congénita y patología placentaria. La RCIU se ha considerado como causa principal de MF en publicaciones con clasificaciones convencionales y su frecuencia oscila entre $8.1 \%$ (11) y $49 \%$ (43), estimándose innecesario el conocimiento de la condición obstétrica originaria de la RCIU. En esta serie la RCIU se presentó en el $49.7 \%$ de los casos y en su mayoría $93.7 \%$, fue secundaria a condición obstétrica específica, como patología placentaria, $\mathrm{SH}$, anomalía congénita, las más frecuentes. La asfixiahipoxia fetal en este estudio se identificó raramente $(0.4 \%)$, y sólo en el parto de término asistido deficientemente, porque las otras causas de asfixia fueron clasificadas en las diferentes patologías obstétricas que la originaron.

El nacimiento prematuro en este trabajo representó el $80 \%$ de los casos de MF y constituyó la situación más importante asociada con la mortinatalidad. Dos tercios de los PP fueron tempranos (menor de 34 semanas) y ocurrieron por diferentes condiciones obstétricas, siendo la IBA la más frecuente $(13,16,19)$. El hallazgo placentario de corioamnionitis y principalmente de funisitis en estos casos confirma la presencia inequívoca de invasión microbiana de la cavidad amniótica (IMCA) y evidencia contundente de la participación de la IBA (47). Esta condición de MF, está descrita ocasionalmente en las clasificaciones convencionales. La importancia de señalarla como causa originaria de MF es por su elevada frecuencia en hospitales públicos de Chile.

El logro de este trabajo, aumento notable de las causas de MF explicables y por consiguiente disminución de las inexplicables, está en la línea que proponen actualmente Recode, Goldenberg y Dudley, et al $(10,17,18)$, que recomiendan usar el método que permite identificar la principal causa obstétrica de la MF (aquélla que en su ausencia no ocurre el óbito) y la secuencia de eventos que llevan a la muerte, porque probablemente constituye la mejor forma de prevenir este resultado adverso.

El método CORM usa como pilar fundamental el estudio histológico placentario, mencionado en aisladas clasificaciones actuales (4,5,9-11), que permite identificar, la mayoría de las veces lo condición obstétrica relevante que lleva a la $\operatorname{MF}(12,13,16,19)$. Los hallazgos de la autopsia fetal en estos casos suelen ser no tan específicos $(44,45)$. Además la autopsia fetal tiene otros inconvenientes, se hace excepcionalmente en algunos países (46), por falta de recursos y especialistas o por la negativa frecuente de la familia y además no modifica la proporción de nacidos muertos inexplicables $(4,44,45)$.

El sistema CORM con el conocimiento de la mayoría de las causas de MF, permite elaborar guías y normas de prevención por causa $(13,16,19)$, que en el futuro constituirán la base para disminuir la MF $(5,10,11)$ y posiblemente el parto prematuro y la muerte neonatal precoz del niño prematuro(48) .

\section{CONCLUSIONES}

Recomendamos el uso del método clasificación de mortinatos CORM, por sus beneficios. Es más eficiente que los métodos convencionales porque identifica la condición obstétrica relevante de la MF y las condiciones responsables de RCIU, del PP y de asfixia. Es reproducible, requiere estudio histopatológico de la placenta y no de autopsia fetal. Permite elaborar normas de prevención de la MF. Puede usarse en países con bajos recursos.

\section{REFERENCIAS}

1. Blencowe $H$, Cousens $S$, Jassir FB, Say L, Chou $\mathrm{D}$, Mathers $\mathrm{C}$, et al. National, regional, and worldwide estimates of stillbirth rates in 2015, with trends from 2000: a systematic analysis. Lancet Glob Health. 2016;4(2): e98-e108. 
2. Lawn JE, Blencowe $H$, Waiswa $P$, Amouzou A, Mathers $C$, Hogan $D$ et al. Stillbirths: rates, risk factors, and acceleration towards 2030. Lancet. 2016; 387:587-603.

3. GBD 2015 Child Mortality Collaborators. Global, regional, national, and selected subnational levels of stillbirths, neonatal, infant, and under-5 mortality, 1980-2015: a systematic analysis for the Global Burden of Disease Study 2015. Lancet. 2016; 388(10053):1725-1774.

4. Korteweg FJ, Gordigin SJ, Timmer A, Erwich JJ, Bergman KA, Bouman $\mathrm{K}$, et al. The Tulip classification of perinatal mortality: Introduction and multidisciplinary inter-rater agreement. $\mathrm{Br} \mathrm{J}$ Obstet Gynaecol 2006; 113: 393-401.

5. Wigglesworth JS. Monitoring perinatal mortality A pathophysiological approach. Lancet 1980; ii: 684-686.

6. De Galan-Roosen AEM, Kuijpers JC, van der Straaten PJC, Merkus JMWM. Fundamental classification of perinatal death; Validation of a new classification system of perinatal death. Eur $\mathrm{J}$ Obstet Gynaecol Reprod Biol 2002; 103: 30-36.

7. Vergani P, Cozzolino S, Pozzi E, Cuttin MS, Greco $M$, Ornaghi $S$, et al. Identifying the causes of stillbirth; a comparison of four classification systems. Am J Obstetrics Gynaecol 2008; 199: 319.e1-319.e4.

8. Hey EN, LLoyd DJ, Wigglesworth JS. Classifying perinatal death: fetal and neonatal factors. $\mathrm{Br} J$ Obstet Gynaecol 1986; 93:1213-1223.

9. Cole SK, Hey EN, Thomson AM. Classifying perinatal death: an obstetric approach. Br J Obstet Gynaecol 1986; 93:1204-1212.

10. Gardosi J, Kady SM, McGeown P, Francis A, Tonks A. Classification of stillbirth by relevant condition at death (ReCoDe): Population based cohort study. BMJ 2005; 331: 1113-1117.

11. Chan A, King JF, Flenady V, Haslam RH, Tudehope DI. Classification of perinatal deaths; development of the Australian and New Zealand classifications. J Paediatr Child Health 2004; 40: 340-347.
12. Ovalle A, Kakarieka E, Correa A., Vial MT, Aspillaga C. Estudio anátomo-clínico de las causas de muerte fetal. Rev Chil Obstet Ginecol 2005; 70: 303-312.

13. Ovalle A, Kakarieka E, Díaz M, García Huidobro T, Acuña MJ, Morong K, et al. Mortalidad perinatal en el parto prematuro entre 22 y 34 semanas en un hospital público de Santiago, Chile. Rev Chil Obstet Ginecol 2012; 77: 263-270

14. Ariff S, Lee AC, Lawn J, Bhutta ZA. Global burden, epidemiologic trends, and prevention of intrapartum related deaths in low-resource settings. Clin Perinatol. 2016;43(3):593-608.

15. Blencowe H, Cousens S, Chou D, et al. Born too soon: the global epidemiology of 15 million preterm births.Reprod Health. 2013;10(Suppl1): S2.

16. Ovalle A, Kakarieka E, Rencoret G, Fuentes A, del Río MJ, Morong $\mathrm{C}$, et al. Factores asociados con el parto prematuro entre 22 y 34 semanas en un hospital público de Santiago. Rev Med Chile 2012; 140:19-29

17. Goldenberg RL, Muhe L, Saleem S, Dhaded S, Goudar SS, Patterson J, et al. Criteria for assigning cause of death for stillbirths and neonatal deaths in research studies in low-midle income countries. J Matern Fetal Neonatal Med. 2018; 23:1-9

18. Dudley DJ, Goldenberg R, Conway D, Silver RM, Saade GR, Varner MW, et al; Stillbirth Research Collaborative Network. A new system for determining the causes of stillbirth. Obstet Gynecol. 2010 Aug;116(2 Pt 1):254-260.

19. Ovalle A, Fuentes A, Chacón V, Espinoza C, González R, Kakarieka $\mathrm{E}$ et al. Método de clasificacion de mortinatos según condición obstetrica relevante de la muerte fetal, en un hospital público de Chile (Método CORM) Rev Med Chile 2016; 144: 1020-1028

20. Pittaluga PE, Díaz AV, Mena NP, Corvalán S. Curva de crecimiento intrauterino para prematuros entre 23 a 36 semanas de edad gestacional. Rev Chil Pediatr 2002; 73: 135-41 ISSN 0370-4106. 
21. Alarcón J, Alarcón Y, Hering E, Buccioni R. Curvas antropométricas de RN chilenos. Rev Chil Pediatr 2008; 79: 364-372.

22. Redline RW. Placental Pathology: A systematic approach with clinical correlations. Placenta 2008; 29 Supple A: S86-91.

23. Kovo M, Schreiber L, Ben-Haroush A, Wand S, Golan A, Bar J. Placental vascular lesion differences in pregnancy induced hypertension and normotensive fetal growth restriction. Am J Obstet Gynecol 2010; 202: 561.e1-5.

24. Huynh J, Dawson D, Roberts D, Bentley-Lewis R. A systematic review of placental pathology in maternal diabetes mellitus. Placenta. 2015; $36: 101-114$

25. Huang L, Liu J, Feng L, Chen Y, Zhang J, Wang W. Maternal prepregnancy obesity is associated with higher risk of placental pathological lesions. Placenta.2014;35:563-569.

26. Ovalle A, Barriga T, Kakarieka E ¿Se relaciona la obesidad en el embarazo con muerte fetal por insuficiencia placentaria? Rev Chil Obstet Ginecol 2017; 82(6):614-620

27. Pinto SM, Dodd S, Walkinshaw SA, Siney C, Kakkar P, Mousa HA. Substance abuse during pregnancy: effect on pregnancy outcomes. Eur $\mathrm{J}$ Obstet Gynecol Reprod Biol 2010; 150: 137-141.

28. Wang N, Tikellis G, Sun C, Pezic A, Wang L, Wells $\mathrm{JC}$, et al. The effect of maternal prenatal smoking and alcohol consumption on the placenta-to-birth weight ratio. Placenta. 2014 Jul;35(7):437-441.

29. Cavallasca JA, Laborde HA, Ruda-Vega H, Nasswetter GG. Maternal and fetal outcomes of 72 pregnancies in Argentine patients with systemic lupus erythematosus (SLE). Clin Rheumatol 2008; 27: 41-46.

30. Geenes VL, Lim YH, Bowman N, Tailor H, Dixon $\mathrm{PH}$, Chambers J, et al. A placental phenotype for intrahepatic cholestasis of pregnancy. Placenta. 2011 Dec;32(12):1026-1032

31. Ugarte L, Cuadra M, Iñaki L, Lapuente O, Gonzalez J. Hemolytic disease of the fetus due to
anti-M antibodies treated with fetal intravenous immunoglobulin therapy. Progr Obstet Ginecol 2015;58 (7): 327-329

32. Simchen MJ, Ofir K, Moran O, Kedem A, Sivan E, Schiff E. Thrombophilic risk factors for placental stillbirth. Eur J Obstet Gynecol Reprod Biol. 2010; 153:160-164.

33. Bernischke K, Kaufman P. Pathology of the human placenta. Chapter: Histopathological Approach to Villous Alteration, 419-434. Chapter: Infectious diseases. New York NY: Springer Verlag NY, Inc; 2001; 591-659.

34. Sebire NJ, Goldin RD, Regan L. Histological chorioamnionitis in relation to clinical presentation at $14-40$ weeks of gestation. J Obstet Gynaecol 2001; 21: 242-245.

35. Al-Adnani M, Sebire NJ. The role of perinatal pathological examination in subclinical infection in obstetrics. Best Pract Res Clin Obstet Gynaecol 2007; 21: 505-521. Review.

36. Ovalle A, Gómez R, Martínez MA, Kakarieka E, Fuentes A, Aspillaga C, et al. Invasión microbiana de la cavidad amniotica en la rotura de membranas de pretérmino. Resultados maternoneonatales y patología placentaria según microorganismo aislado. Rev Med Chile 2005; 133: 51-61.

37. Park CW, Moon KC, Park JS, Jun JK, Romero R, Yoon BH. The Involvement of Human Amnion in Histologic Chorioamnionitis is an Indicator that a Fetal and an Intra-Amniotic Infl ammatory Response is More Likely and Severe: Clinical Implications Placenta 2009; 30: 56-61.

38. Offenbacher S, Boggess KA, Murtha AP, Jared $\mathrm{HL}$, Lieff S, Mckaig RG, et al. Progressive periodontal disease and risk of very preterm delivery. Obstet Gynecol 2006;107: 29-36.

39. Fox H. Pathology of the Placenta. Chapter: Histological Abnormalities of the Placenta. Philadelphia, Pa: WB Saunders Co; 1978; 149-197

40. Keeling JW. Fetal and Neonatal Pathology. Chapter: Placenta and Umbilical Cord. Berlin Heidelgerg: Spriger-Verlag. 1987; 45-67. 
41. Perrin VDK. Pathology of the Placena. Chapter: Placenta as a Reflection of maternal disease. New York: Churchill-Livingstone Inc; 1984; 57-70

42. Hecht JL, Allred EN, Kliman HJ, Zambrano E, Doss BJ, Husain A, et al. Elgan Study Investigators. Histological characteristics of singleton placentas delivered before the 28th week of gestation. Pathology 2008; 40: 372-376.

43. Ego A, Zeitlin J, Batailler P, Cornec S, Fondeur A, Baran-Marszak $M$, et al. Stillbirth classification in population-based data and role of fetal growth restriction: the example of RECODE. BMC Pregnancy Childbirth.2013 Oct 3;13:182

44. Man J, Hutchinson JC, Heazell AE, Ashworth M, Levine S, Sebire NJ. Stillbirth and intrauterine fetal death: factors affecting determination of cause of death at autopsy. Ultrasound Obstet Gynecol. 2016;48(5):566-573.
45. Pasztor N, Kereszturi A, Kozinszky Z, Pál A. Identification of causes of stillbirth through autopsy and placental examination reports. Fetal Pediatr Pathol. 2014;33(1):49-54.

46. Kaschula RO. The pediatric autopsy in Africa. Arch Pathol Lab Med. 2013;137(6):756-766

47. Ovalle A, Martínez MA, Kakarieka ME, Gómez R, Torres J, Fuentes A, et al. Histopatología de la placenta en la rotura prematura de membranas de pretérmino. Relación con la microbiología aislada y con los resultados maternoneonatales. Rev Med Chil 1998; 126: 930-942.

48. Ovalle A, Martínez MA, Figueroa J. ¿Se puede prevenir el parto prematuro por infección bacteriana ascendente y sus resultados adversos en los hospitales públicos de Chile? Artículo de revisión. Presentado para publicación. 
Tabla 1. Clasificación de la muerte fetal. Método CORM

(Condición obstétrica relevante u originaria de la muerte). Ovalle y cols.

\begin{tabular}{|c|c|}
\hline Condición & \\
\hline \multicolumn{2}{|l|}{ A.Materna } \\
\hline \multirow[t]{8}{*}{ Enfermedad } & 1.SH \\
\hline & 2.Diabetes \\
\hline & 3.Obesidad \\
\hline & 4.Consumo drogas \\
\hline & 5.Lupus eritematoso sistémico \\
\hline & 6.CIE \\
\hline & 7.Isoinmunización \\
\hline & 8.Otras \\
\hline \multicolumn{2}{|l|}{ Infección } \\
\hline \multirow[t]{2}{*}{ Ascendente } & 9.Bacteriana \\
\hline & 10.Micótica \\
\hline \multirow[t]{5}{*}{ Transplacentaria } & 11.Viral \\
\hline & 12.Sífilis \\
\hline & 13.Listeria monocytogenes \\
\hline & 14.Enfermedad periodontal \\
\hline & 15.Otras \\
\hline \multicolumn{2}{|l|}{ B.Fetal } \\
\hline \multirow[t]{2}{*}{ Anomalía congénita } & 1.Cromosómica \\
\hline & 2.No cromosómica \\
\hline $\mathrm{RCIU}$ & 3. De condición no precisada \\
\hline Hidrops & 4. De condición no precisada \\
\hline \multirow[t]{2}{*}{ Embarazo múltiple (monocorial biamniótico) } & 5.Síndrome de transfusión feto-fetal \\
\hline & 6.RCIU selectiva \\
\hline \multicolumn{2}{|l|}{ C.Placentaria } \\
\hline \multirow[t]{5}{*}{ Patología Placentaria } & 1.DPPNI primario \\
\hline & $\begin{array}{l}\text { 2. Alteraciones circulatorias maternas y fetales y } \\
\text { RCIU }\end{array}$ \\
\hline & 3.Deciduitis, vellositis crónica y RCIU \\
\hline & 4.Otras y RCIU \\
\hline & 5.Placenta previa \\
\hline Patologías de Cordón & $\begin{array}{l}\text { 6. Circular, nudo, trombosis y/o ruptura, } \\
\text { hiperrotación, procidencia, hematoma, inserción } \\
\text { velamentosa }\end{array}$ \\
\hline \multicolumn{2}{|l|}{ D.Uterina } \\
\hline & $\begin{array}{l}\text { 1.Malformación uterina, trauma parto, parto } \\
\text { provocado, rotura uterina }\end{array}$ \\
\hline \multicolumn{2}{|l|}{ E.Asfixia durante el parto de término } \\
\hline F. No clasificable, No explicable & \\
\hline
\end{tabular}

$\mathrm{SH}$, sídrome hipertensivo; CIE, colestasia intrahepática del embarazo; RCIU, restricción del crecimiento fetal intrauterino; DPPNI, desprendimiento prematuro de placenta normoinsrta. 
Figura 1. Tasas de mortalidad Fetal total y por nacionalidad de las madres. HCSBA período 2008-2017

Tasa de MF $\mathrm{x}$

1000 nacidos




Tabla 2. Condición obstétrica relevante de la muerte fetal Método CORM, período 2008-2017. Hospital Clínico San Borja Arriarán

\begin{tabular}{|l|c|c|}
\hline \multicolumn{1}{|c|}{ Condición obstétrica relevante } & No & $\%$ \\
\hline IBA & 119 & 24.9 \\
\hline Anomalía Congénita & 86 & 18.0 \\
\hline Patología placentaria & 67 & 14.0 \\
\hline SH & 60 & 12.6 \\
\hline Otra Enfermedad materna & 33 & 6.9 \\
\hline Embarazo múltiple & 17 & 3.5 \\
\hline Diabetes & 16 & 3.3 \\
\hline Infección transplacentaria & 15 & 3.1 \\
\hline RCIU de causa no explicable & 15 & 3.1 \\
\hline Obesidad & 14 & 2.9 \\
\hline Patología del cordón & 3 & 0.6 \\
\hline Asfixia intraparto & 2 & 0.4 \\
\hline IMA & 1 & 0.2 \\
\hline No clasificable, No explicable & 31 & 6.5 \\
\hline & 479 & 100.0 \\
\hline
\end{tabular}

IBA, infección bacteriana ascendente; $\mathrm{SH}$, síndrome hipertensivo;

IMA, infección micótica ascendente

$\mathrm{RCIU}$, restricción del crecimiento fetal intrauterino

RCIU total $49.7 \%(238 / 497)$

RCIU secundaria a condición obstétrica 93.7\% (223/238); 6,3\% condición no explicable 
Tabla 3. Características de la población en estudio. Muertes fetales. Clasificación según método CORM, período 2008-2017. Hospital Clínico San Borja Arriarán.

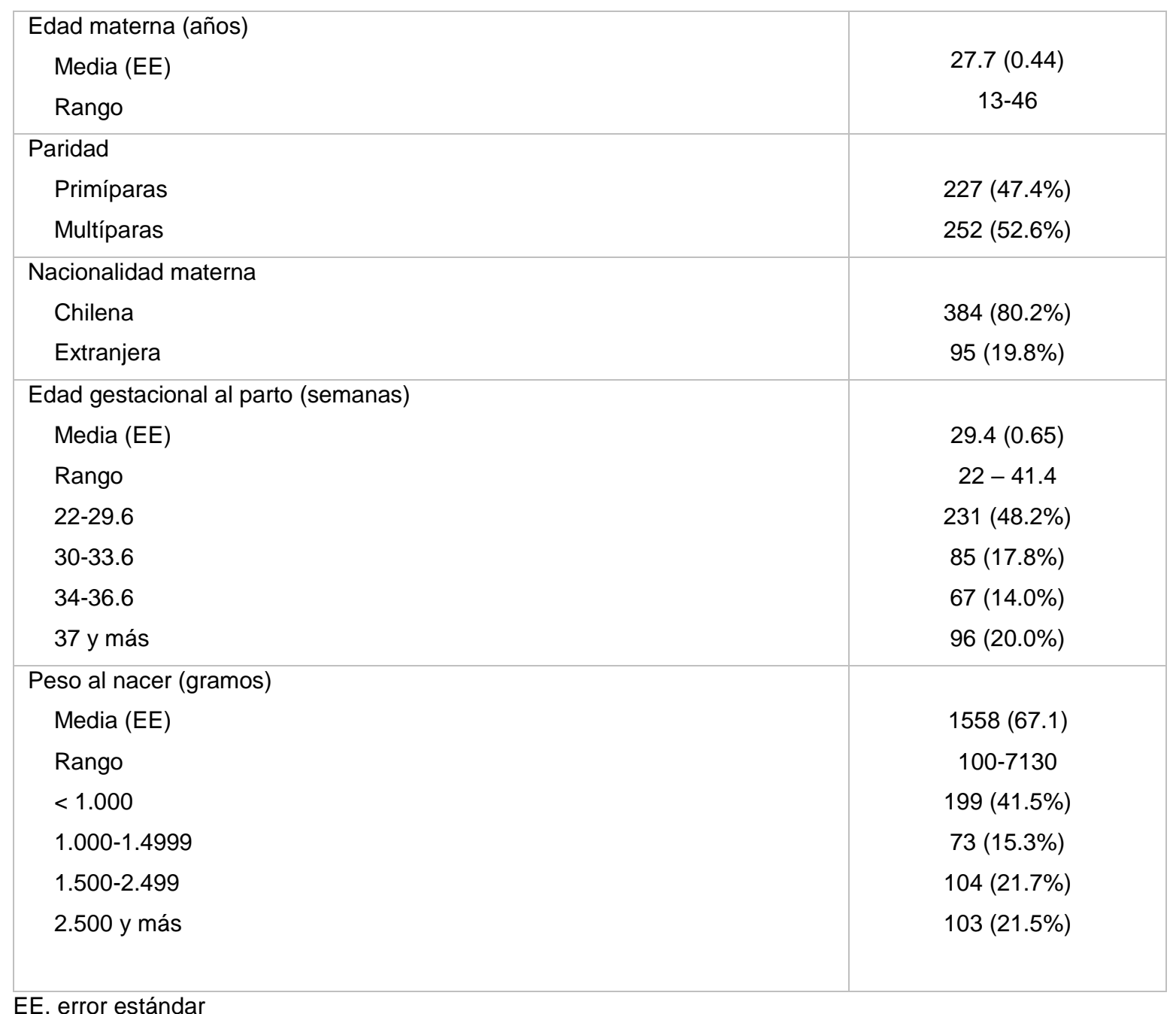

